\title{
IMMUNOHISTOCHEMICAL EXPRESSION OF BRAFV600E IN MELANOMA CASES
}

\author{
Muhammad Umer Ch, Farhan Akhtar*, Nighat Jamal, Rabia Ahmad, Sumaira Bukhsh**, Hamza Mansur*** \\ Pakistan Naval Ship Shifa Hospital, Karachi Pakistan, *Pak Emirates Military Hospital/National University of Medical Sciences (NUMS) Rawalpindi Pakistan, \\ ${ }^{* *}$ Combined Military Hospital Malir/National University of Medical Sciences (NUMS) Pakistan, ${ }^{* * *}$ Armed Forces Institute of Pathology/ \\ National University of Medical Sciences (NUMS) Rawalpindi Pakistan
}

\begin{abstract}
Objective: To evaluate the diagnostic value of Immunohistochemistry (IHC) for finding BRAFV600E mutation in melanoma patients.

Study Design: Cross-sectional study.

Place and Duration of Study: Department of Histopathology, Armed Forces Institute of Pathology, Rawalpindi Pakistan, Jan 2018 to Apr 2019.

Methodology: Hundred histologically confirmed cases of melanoma aged between 18-80 years were included over a period of one year. To create the tissue microarray and to conduct immunohistochemistry with VE1 antibody melanoma lesion samples were selected.

Results: Ninety-four cases out of 100, were melanoma positive for BRAFV600E detected on immunohistochemistry (IHC) using a monoclonal antibody. The skin was the primary site in seventy-nine (79\%) patients, unknown (metastatic) in nineteen and uveal was only in two cases. The diagnostic significance of immunohistochemistry (IHC) for detecting the BRAFV600E mutation in melanoma patients was independent of age, site and stage at diagnosis ( $p$-value $<0.05)$.

Conclusion: Testing of BRAV600E mutation by immunohistochemistry is a rapid, reliable and cost-effective tool in melanoma patients which may further help clinicians in initiating targeted therapy for these patients.
\end{abstract}

Keywords: BRAFV600E mutation, Immunohistochemistry, Monoclonal antibody, Malignant melanoma.

How to Cite This Article: Ch MA, Akhtar F, Jamal N, Ahmad R, Buksh S, Mansur H. Immunohistochemical Expression of BRAFV600E in Melanoma Cases. Pak Armed Forces Med J 2021; 71(5): 1885-1888. doi: https://doi.org/10.51253/pafmj.v71i5.5955

This is an Open Access article distributed under the terms of the Creative Commons Attribution License (https://creativecommons.org/licenses/by-nc/4.0/), which permits unrestricted use, distribution, and reproduction in any medium provided the original work is properly cited.

\section{INTRODUCTION}

The worldwide incidence of melanoma has steadily increased over the last several decades. The prevalence varies by race: 1.9/100,000 in Hispanics 9.2/ 100,000 in whites, and $0.7 \%$ to $1.2 / 100,000$ in blacks and Asian ${ }^{1}$. In spite of significant advancements in medicine, melanoma remains a huge burden on the healthcare system in developing countries due to the substantial annual costs incurred for its management ${ }^{2}$.

Melanoma is a difficult carcinoma to cure ranking the fifth and seventh most frequent tumour diagnosed in males and females respectively ${ }^{3}$. In malignant melanoma, activating BRAF mutations are identified at a high rate. More than $90 \%$ of such mutations are characterized by glutamate to valine replacement at location 600 (V600E), leading to a substantial rise in protein kinase activity. Especially in primary and metastatic melanoma (20-70\%), papillary thyroid tum-our (40$70 \%$ ), colorectal cancer (5-10\%), and selected benign tumours like melanocytic nevi ${ }^{4}$, these mutations are seen at increased levels. Because of its incidence and

Correspondence: Dr Muhammad Umer Ch, Department of Histopathology, PNS Shifa Hospital, Karachi-Pakistan

Received: 30 Dec 2020; revision received: 02 Mar 2021; accepted: 10 Mar 2021 contribution in raising tumour growth and metastases, BRAFV600E has been recognized as an effective biomarker for clinicians ${ }^{5}$.

DNA based techniques like direct DNA sequencing and allele-specific PCR are the gold standard to detect BRAF mutations in clinical settings. The most common ones are the amplification refractory mutation system-PCR, classical Sanger sequencing, highresolution melting, ligase detection reaction, pyrosequencing MassArray and next-generation sequencing (NGS) ${ }^{6}$.

Though these techniques for mutational analysis are accurate and reliable, they are expensive and timeconsuming. The genetic analysis becomes less feasible because of its relatively high labour cost, costly equipment, technological flaws, complex analysis and the need for a genetic testing specialist and often not regularly accessible in all anatomic pathology laboratories ${ }^{7}$.

The production of new antibodies directed against V600E antigen has lately, unlocked the way to a simpler but more successful technique for the detection of this mutation and now is available commercially from Spring Bioscience and Ventana ${ }^{8,9}$. The first BRAFV600E -specific antibody (VE1) was developed 
by Feller et al, which has validated to be very sensitive and precise for recognizing this alteration in melanoma cases ${ }^{10}$ and have paved the way to use IHC, which is a relatively quicker, less costly and more accessible technique for evaluating the BRAF status in the biopsy specimen.

In view of the above, this study was designed to validate the diagnostic significance of IHC in detecting the BRAFV600E mutation in melanoma patients. Currently, the study is prospective using a relatively larger sample size which would be a valuable contribution to the already existing pool of knowledge.

\section{METHODOLOGY}

The cross sectional study was approved by the institutional review board of Armed Forces Institute of Pathology [FC-READ-IRB/18/885] from January 2018 to April 2019.

Inclusion Criteria: The histologically confirmed cases of melanoma aged between 18-85 years of either gender were included using non-probability consecutive sampling technique.

Exclusion Criteria: The patients having prior chemotherapy, antiangiogenic therapy and radiotherapy were excluded.

The sample size was calculated by using OpenEpi calculator by taking the prevalence of BRAFV 600E mutation on immunohistochemistry in melanoma patients i.e. $94 \% 8$, margin of error $=4 \%$, confidence level= $95 \%$, then calculated sample size was 100 . Clinicopathological features such as gender, age, site, stage, distant and nodal metastasis were noted.

Four-micrometer thick slices were cut on microtome from formalin-fixed, paraffin-embedded (FFPE) tissue blocks. Testing was performed with the Benchmark ULTRA platform with pre-peroxidase inhibition and primary antibody incubation for $15 \mathrm{~min}$ at $37^{\circ} \mathrm{C}$ using anti V600 E monoclonal primary antibody (Ventana Medical Systems, Inc Cat. Number 790-4855). Anti-body's final concentration was $\sim 12 \mu \mathrm{g} / \mathrm{ml}$. To visualize the BRAF expression diaminoben-zidine staining was used. Hematoxylin II and Bluing Reagent were used for counter staining the tissues for $4 \mathrm{~min}$. Negative control in the form of mouse monoclonal antibody was also applied.

Ultimately, specimens after immunohisto-chemistry were seen by autonomous double-blinded analysts who didn't have cellular and laboratory-based patient's records. In most cases, the analyses of the two observers were comparable and re-examination as well as consensus resolved few disparities. In positive tumor cells, the VE1 antibody demonstrates cytoplasmic staining in accordance with Boursault et all1. Immunostaining was generally defined as positive or negative. When $>90 \%$ of cancer cells displayed distinct moderate to strong brown intracellular discoloration they were taken as positive, whereas when there was no discoloration, only nuclear stain, focal staining of tumor cellsor staining of lymphocytes, monocytes/macrophagesthey were considered negative. Furthermore, the immunostaining intensity was graded 0 if no discolouration was observed, Grade 1 if there was weakcytoplasmic discoloration, moderate diffuse cytoplasmic staining was given grade 2 and grade 3 was alloted with strongmainly granular intracellular brown discoloration. No staining (grade 0) and grade 1 staining were considered negative for $\mathrm{V} 600 \mathrm{E}$, whereas grade 2 and grade 3 were considered as positive as per Løes et al12.

Statistical software SPSS-25 was used to analyze the data. Median (range) was computed for quantitative variables like age as the assumption of normality was violated. Frequencies and percentages were computed for qualitative data like gender, site, stage, distant and nodal metastasis along with outcome variables i.e. BRAFV600E detected by immunohistochemistry using a monoclonal antibody in melanoma patients. Effect modifiers were controlled through the stratification of age, gender, site, stage, distant and nodal metastasis. chi-square test was applied post stratification. The $p$-value of $<0.05$ was considered statistically significant.

\section{RESULTS}

Out of the total hundred patient samples range for age in this research was 18-80 years with a median age of 63 (24-85). Fifty-nine (59\%) were male and 41 (31\%) were female. The skin was the primary site in seventy-nine $(79 \%)$ patients, unknown (metastatic) in nineteen $(19 \%)$ and uveal was only in two $(2 \%)$ cases. Seventeen $(17 \%)$ of the patients had stage I disease at diagnosis, thirty-four (34\%) patients were at stage II, forty-three $(43 \%)$ were at stage III and six $(6 \%)$ were at stage IV. According to the melanoma staging system of the American Joint Committee on Cancer (AJCC), twenty-three $(23 \%)$ patients had M1a disease, nineteen (19\%) patients had M1b disease and fifty-eight (58\%) patients had M1c stage, shown in the Table-I.

Nighty four cases of Melanoma were positive for BRAF V600E detected on immunohistochemical (IHC) method using a monoclonal antibody, shown in Table II. 
Table-I: Demographic details of the patients.

\begin{tabular}{|c|c|}
\hline Parameters & n (\%) \\
\hline \multicolumn{2}{|l|}{ Gender } \\
\hline Male & $59(59 \%)$ \\
\hline Female & $41(31 \%)$ \\
\hline \multicolumn{2}{|c|}{ Site of Melanoma } \\
\hline Skin & $79(79 \%)$ \\
\hline Unknown & $19(19 \%)$ \\
\hline Uveal & $02(2 \%)$ \\
\hline \multicolumn{2}{|c|}{ Stage at Diagnosis } \\
\hline I & $17(17 \%)$ \\
\hline II & $34(34 \%)$ \\
\hline III & $43(43 \%)$ \\
\hline IV & $6(6 \%)$ \\
\hline \multicolumn{2}{|c|}{ Distant Metastasis } \\
\hline M1a & $23(23 \%)$ \\
\hline M1b & $19(19 \%)$ \\
\hline M1c & $58(58 \%)$ \\
\hline \multicolumn{2}{|c|}{ Nodal Metastasis } \\
\hline$<2$ & $49(49 \%)$ \\
\hline$>2$ & $51(51 \%)$ \\
\hline
\end{tabular}

Table-II: Frequency of BRAF V600E detected on immunohistochemical (IHC) method using amonoclonal antibody in melanoma patients.

\begin{tabular}{l|c}
\hline BRAF V600E on IHC & n (\%) \\
\hline Positive & $94(94 \%)$ \\
Negative & $06(6 \%)$ \\
\hline
\end{tabular}

Table-III: Association between clinicopathological features and frequency of BRAFV600E detected immunohistochemical method using monoclonal antibody in melanoma patients.

\begin{tabular}{|c|c|c|c|}
\hline \multirow{2}{*}{$\begin{array}{l}\text { Demographic } \\
\text { Details }\end{array}$} & \multicolumn{2}{|c|}{$\begin{array}{c}\text { BRAF V600E on } \\
\text { Immunohistochemistry }\end{array}$} & \multirow{2}{*}{$\begin{array}{c}p- \\
\text { value }\end{array}$} \\
\hline & Positive, n(\%) & Negative, $n(\%)$ & \\
\hline \multicolumn{3}{|l|}{ Age } & \multirow{3}{*}{0.002} \\
\hline $18-40$ years & $08(8 \%)$ & $04(4 \%)$ & \\
\hline 40 years & $86(86 \%)$ & $02(2 \%)$ & \\
\hline \multicolumn{3}{|l|}{ Gender } & \multirow{3}{*}{0.224} \\
\hline Male & $57(57 \%)$ & $02(2 \%)$ & \\
\hline Female & $37(37 \%)$ & $04(4 \%)$ & \\
\hline \multicolumn{3}{|c|}{ Site of Melanoma } & \multirow{4}{*}{0.03} \\
\hline Skin & $75(75 \%)$ & $04(4 \%)$ & \\
\hline Unknown & $18(18 \%)$ & $01(1 \%)$ & \\
\hline Uveal & $01(1 \%)$ & $01(1 \%)$ & \\
\hline \multicolumn{3}{|c|}{ Stage at Diagnosis } & \multirow{5}{*}{$<0.001$} \\
\hline $\mathrm{I}$ & $16(16 \%)$ & $01(1 \%)$ & \\
\hline II & $33(33 \%)$ & $01(1 \%)$ & \\
\hline III & $40(40 \%)$ & $03(3 \%)$ & \\
\hline IV & $05(5 \%)$ & $01(1 \%)$ & \\
\hline \multicolumn{3}{|c|}{ Distant Metastasis } & \multirow{4}{*}{0.825} \\
\hline M1a & $21(21 \%)$ & $02(2 \%)$ & \\
\hline M1b & $18(18 \%)$ & $01(1 \%)$ & \\
\hline M1c & $55(55 \%)$ & $03(3 \%)$ & \\
\hline \multicolumn{3}{|c|}{ Nodal Metastasis } & \multirow{3}{*}{0.678} \\
\hline$<2$ & $47(47 \%)$ & $02(2 \%)$ & \\
\hline$>2$ & $47(47 \%)$ & $04(4 \%)$ & \\
\hline
\end{tabular}

Multivariate analysis showed that the age, site of Melanoma and stage at diagnosis are independent factors ( $p$-value <0.05). However, the same may be affected with gender, distant and nodal metastasis, shown in the the Table-III.

\section{DISCUSSION}

In the era of personalized medicine use of immunohistochemistry as a prognostic and predictive marker is on the rise, since it is a comparatively quick and economical method that doesn't necessitate large tumour cell substance ${ }^{13}$. It is also simple to perform and is regarded to be extremely sensitive and precise as the monoclonal antibodies of a specific genetic alteration are typically focused towards the epitope of beneficial concern ${ }^{14}$.

Regarding melanoma, few studies are available on the comparison of the diagnostic utility of clone VE1 in detecting BRAF V600E mutation with those of other molecular methodologies. Moreover, the clinical characteristics of patients with melanoma BRAFV600E mutation are not consistent across different studies ${ }^{15,16}$. The emphasis of this research wasn't on assessing the incidence of BRAFV600E mutations in melanoma, but to discover the likelihood of employing a monoclonal VE1 antibody immunohistochemistry test as a substitute for currently used molecular methodologies in identifying BRAF V600E mutation in melanoma. Hence, our cases were not chosen particularly to validate mutation rates.

We found BRAF V600E mutations were positive in $94 \%$ of the patients. Our results are almost like those of Schirosi et al17, who studied 64 histologically confirmed metastatic cases using the fully automatized immuno-histochemistry method, reached a positive predictive value (PPV) of $97.1 \%$. Similarly, our results are also the same as that of Yaman et al17, who studied 48 specimens including both the primary and metastatic melanoma biopsies using the fully automatized immunohistochemical technique with supplementation of an amplification reagent kit, reported a positive predictive value of $98.2 \%$ \& negative predictive value of $89.7 \%$. Another study close to our results is Haung et al18 who in a study of 73 cases of melanoma from lymph nodes and metastatic sites using the bond max auto-stainer showed the sensitivity and specificity of $100 \%$ and $98.6 \%$ respectively with an overall concordance of $96 \%$ between immuno-histochemical and direct sequencing method. Similarly, Manfredi et al. Have mentioned highly concordant results between immunohistochemistry and molecular testing of mutated BRAFV600E in primary and metastatic melanoma ${ }^{19}$. 
It should, however, be acknowledged that our observed prevalence of BRAFV600E in melanoma is significant, which is a precedent. Researches on the incidence of BRAF mutations in melanoma signify that they're often seen in melanomas of skin with a comparatively high prevalence observed in those who are sporadically subjected to sunlight versus continuously subjected to sunlight ${ }^{6}$. As a greater percentage of our cases were from continuously sun-exposed sites, this could account for the higher frequency of BRAFV 600E throughout the current cohort. The results of this study, therefore, shed light on the possible use of IHC with the anti-BRAF (V600E) antibody as an additional evaluation tool for assessing the status of BRAFV600E mutation in melanoma.

With regards to the association between clinicopathological features and immunohistochemistry results, age, site and stage at the time of diagnosis were significantly associated with BRAFV600E mutation. Our findings are consistent with literature reporting that the BRAF mutation has been correlated with younger patients ${ }^{20}$. Additional studies on a larger cohort, multicentre, standardized and prospective studies are essential to authenticate our results and for immunohistochemistry to supplement or substitute DNA-based techniques.

\section{CONCLUSION}

The findings of our study allow the use of immunohistochemistry as a cost-effective, rapid and reliable diagnostic tool in detecting the BRAFV600E mutations in melanoma patients. This would help the clinicians in initiating the personalized medicine tailored to the needs of individual patients timely. However, more studies with a larger sample size are needed in order to draw a robust conclusion regarding the efficacy of immunohistochemistry in detecting the BRAFV600E mutations in melanoma patients.

\section{Conflict of Interest: None.}

\section{Authors' Contribution}

MUC: Direct contribution, conception, data analysis. FA: Manuscript preparation. NJ: Data analysis. RA: Intellectual contribution to the analysis. SB: Data collection. HM: Intellectual contribution to interpretation.

\section{REFERENCES}

1. Matthews NH, Li WQ, Qureshi AA. Epidemiology of Melanoma. In: Ward WH, Farma JM, editors. Cutaneous Melanoma: Etiology and Therapy. Brisbane (AU): Codon Publica 2017, [Internet] https:// pubmed.ncbi.nlm.nih.gov/29461782/

2. Ghasemi M, Larijani LV, Emadian O, Yazdani J, Sajadianfar A, Abediankenari S. Immunohistochemical investigation of mutant BRAF V600E in common pigmented skin neoplasms, study on a sample of Iranian patients. Iran J Pathol 2019; 14(1): 8-16.

3. Jemal A, Siegel R, Xu J, Ward E. Cancer statistics, 2010. CA Cancer J Clin 2010; 60(5): 277-300.
4. Tosuner, Z, Geçer MÖ, Hatiboğlu MA, Abdallah A. BRAF V600E mutation and BRAF VE1 immunoexpression profiles in different types of glioblastoma. Oncol Letters 2018; 16(2): 2402-240.

5. Shinozaki M, Fujimoto A, Morton D. Incidence of BRAF oncogene mutation and clinical relevance for primary cutaneous melanomas. Clin Cancer Res 2004; 10(5): 1753-1757.

6. Ihle MA, Fassunke J, König K, Grünewald I, Schlaak M. Comparison of high resolution melting analysis, pyrosequencing, nextgeneration sequencing and immunohistochemistry to conventional Sanger sequencing for the detection of p.V600E and nonp.V600E BRAF mutations. BMC Cancer 2014; 14(1): 13-18.

7. Rashid FA, Tabussum S, Khan MS, Ansari HR, Asif M, Shaikh AK. VE1 immunohistochemistry is an adjunct tool for detection of BRAF V600E mutation: Validation in thyroid cancer patients. J Clin Lab Anal 2020; 35(2): 1-7.

8. Wilmott JS, Menzies AM, Haydu LE, Capper D, Preusser M, Zhang YE, et al. BRAF(V600E) protein expression and outcome from BRAF inhibitor treatment in BRAF(V600E) metastatic melanoma. Br J Cancer 2013; 108(4): 924-931.

9. Pearlstein MV, Zedek DC, Ollila DW, Treece A, Gulley ML. Validation of the VE1 immunostain for the BRAF V600E mutation in melanoma. J Cutan Pathol 2014; 41(9): 724-732.

10. Lo M, Paterson A, Maraka J, Clark R, Goodwill J, Nobes J, et al. A UK feasibility and validation study of the VE1 monoclonal antibody immunohistochemistry stain for BRAF-V600E mutations in metastatic melanoma. Br J Cancer 2016; 115(2): 223-227.

11. Boursault L, Haddad V, Vergier B, Cappellen D, Verdon S. Tumor homogeneity between primary and metastatic sites for BRAF status in metastatic melanoma determined by immunehistochemicalandmolecular testing. PLoS One 2013; 8(8): e70826.

12. Løes IM, Immervoll H, Angelsen JH, Horn A, Geisler J, Busch C, et al. Performance comparison of three BRAF V600E detection methods in malignant melanoma and colorectal cancer specimens. Tumour Biol 2015; 36(2): 1003-1113.

13. Suh MS, Choi YD, Lee JB, Lee SC, Won YH. Sensitivity and usefulness of VE1 immunohistochemical staining in acral melanomas with BRAF mutation. Ann Dermatol 2018; 30(5): 556-561.

14. Karbel HAE, Ejam SS, Naji AZ. Immunohistochemical Study Using Monoclonal VE1 Antibody Can Substitute the Molecular Tests for Apprehension of BRAFV600E Mutation in Patients with Non-small-Cell Lung Carcinoma. Hindawi 2019; 12(2): 1-6.

15. Hui Liu, Zhongwu Li, Yan Wang, Qin Feng, Lu Si, Chuanliang Cui. Immunohistochemical detection of the BRAF V600E mutation in melanoma patients with monoclonal antibody VE1. Pathol Int 2014; 64(12): 601-606.

16. Schirosi L, Strippoli S, Gaudio F, Graziano G, Popescu O, Guida $\mathrm{M}$. Is immunohistochemistry of BRAF V600E useful as a screening tool and during progression disease of melanoma patients? BMC Cancer 2016; 16(1): 905-910.

17. Yaman B. BRAF-V600 mutation hetero-geneity in primary and metastatic melanoma: a study with pyro-sequencing and immunohistochemistry. Am J Dermatopathol 2016; 38(24): 113-120.

18. Haug WK, Kuo TT, Wu CE, Cheng HY, Hsieh CH, Hsieh JJ, et al. A comparison of immunohistochemical and molecular methods used for analyzing the BRAF V600E gene mutation malignant melanoma in Taiwan. Asia-Pac J Clin Oncol 2019; 12(4): 403-408.

19. Manfredi L, Meyer N, Tournier E, Grand D, Uro-Coste E. Highly concordant results between immuno-histochemistry and molecular testing of mutated V600E BRAF in primary and metastatic melanoma. Acta Derm Venereol 2016; 96(4): 630-634.

20. Ekedahl H, Cirenajwis H, Harbst $K$, Carneiro A. The clinical significance of BRAF and NRAS mutations in a clinic-based metastatic melanoma cohort. Br J Dermatol 2013; 169(5): 10491055. 